\title{
Electrical Homogeneity Mapping of Epitaxial Graphene on Silicon Carbide
}

\begin{abstract}
Whelan, Patrick Rebsdorf; Panchal, Vishal; Petersen, Dirch Hjorth; Mackenzie, David M. A.; Melios, Christos; Pasternak, Iwona; Gallop, John; Østerberg, Frederik Westergaard; Jepsen, Peter Uhd; Strupinski, Wlodek
\end{abstract}

Total number of authors:

12

Published in:

A C S Applied Materials and Interfaces

Link to article, DOI:

10.1021/acsami.8b11428

Publication date:

2018

Document Version

Publisher's PDF, also known as Version of record

Link back to DTU Orbit

Citation (APA):

Whelan, P. R., Panchal, V., Petersen, D. H., Mackenzie, D. M. A., Melios, C., Pasternak, I., Gallop, J., Østerberg, F. W., Jepsen, P. U., Strupinski, W., Kazakova, O., \& Bøggild, P. (2018). Electrical Homogeneity Mapping of Epitaxial Graphene on Silicon Carbide. A C S Applied Materials and Interfaces, 10(37), 3164131647. https://doi.org/10.1021/acsami.8b11428

\section{General rights}

Copyright and moral rights for the publications made accessible in the public portal are retained by the authors and/or other copyright owners and it is a condition of accessing publications that users recognise and abide by the legal requirements associated with these rights.

- Users may download and print one copy of any publication from the public portal for the purpose of private study or research.

- You may not further distribute the material or use it for any profit-making activity or commercial gain

- You may freely distribute the URL identifying the publication in the public portal 


\section{Electrical Homogeneity Mapping of Epitaxial Graphene on Silicon Carbide}

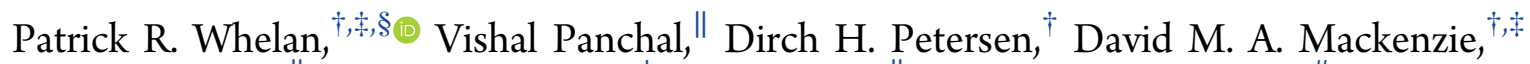
Christos Melios, ${ }^{\circledR \odot}$ Iwona Pasternak, ${ }^{\perp}$ John Gallop, " Frederik W. Østerberg, ${ }^{\#}$ Peter U. Jepsen, ${ }^{\ddagger}, \S$ Wlodek Strupinski, ${ }^{\perp}$ Olga Kazakova, $\|$ and Peter Bøggild ${ }^{*},+,+[0$

${ }^{\dagger}$ DTU Nanotech and ${ }^{\ddagger}$ Center for Nanostructured Graphene (CNG), Technical University of Denmark, Ørsteds Plads 345C, DK-2800 Kongens Lyngby, Denmark

${ }^{\S}$ DTU Fotonik, Technical University of Denmark, Ørsteds Plads 343, DK-2800 Kongens Lyngby, Denmark

"National Physical Laboratory, Hampton Road, Teddington TW11 0LW, U.K.

${ }^{\perp}$ Faculty of Physics, Warsaw University of Technology, Koszykowa 75, 00-662 Warsaw, Poland

${ }^{\#}$ Capres A/S, Diplomvej 373, DK-2800 Kongens Lyngby, Denmark

Supporting Information

ABSTRACT: Epitaxial graphene is a promising route to wafer-scale production of electronic graphene devices. Chemical vapor deposition of graphene on silicon carbide offers epitaxial growth with layer control but is subject to significant spatial and wafer-to-wafer variability. We use terahertz time-domain spectroscopy and micro four-point probes to analyze the spatial variations of quasi-freestanding bilayer graphene grown on 4 in. silicon carbide ( $\mathrm{SiC}$ ) wafers and find significant variations in electrical properties across large regions, which are even reproduced across graphene on different $\mathrm{SiC}$ wafers cut from the same ingot. The dc sheet conductivity of epitaxial graphene was found to vary more
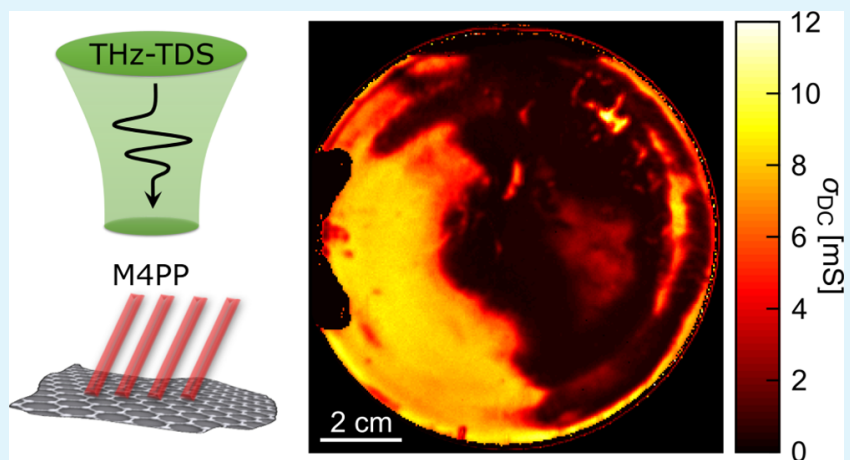
than 1 order of magnitude across a 4 in. SiC wafer. To determine the origin of the variations, we compare different optical and scanning probe microscopies with the electrical measurements from nano- to millimeter scale and identify three distinct qualities of graphene, which can be attributed to the microstructure of the $\mathrm{SiC}$ surface.

KEYWORDS: graphene, SiC, conductivity, terahertz spectroscopy, micro four-point probe, metrology

\section{INTRODUCTION}

Graphene growth on silicon carbide $(\mathrm{SiC})$ by chemical vapor deposition (CVD) combined with hydrogen intercalation allows 4 in. wafers to be covered with quasi-freestanding (QFS) single-layer graphene (1LG) or bilayer graphene (2LG). ${ }^{1-5}$ This technology enhances the carrier mobility and changes the conduction from electrons to holes. ${ }^{2,6}$ Precise control of such properties is extremely useful for applications in integrated circuits, $^{7-9}$ gas sensors, ${ }^{10-12}$ photonics and optoelectronics, $^{13}$ and terahertz electronics. ${ }^{14}$ However, progress on the development for these types of devices on wafer scale has been limited because of the lack of rapid electrical, optical, and structural characterization techniques capable of imaging entire wafers with spatial resolution ranging from millimeters down to tens of nanometers. ${ }^{15,16}$

Electrical characterization of graphene on $\mathrm{SiC}$ has typically required fabrication of Hall bar-type devices, which allow measurements of conductivity, carrier density, mobility, and mean free path. ${ }^{17}$ However, these types of devices are sensitive to geometrical errors and material uniformity and provide values that are the weighted average over the device area, ${ }^{18,19}$ which can range from micrometers to millimeters square, thus making it difficult and cumbersome to carry out high spatial resolution mapping over larger areas because of inherent inhomogeneities in graphene transport properties. $^{20}$ Optical imaging techniques such as optical microscopy and Raman spectroscopy have been successfully used for providing vast information on optical and structural properties, respectively. $^{21,22}$ Confocal laser scanning microscopy (CLSM) is an alternative rapid characterization technique for mapping the optical reflection over large areas, while retaining high spatial resolution of $\sim 150 \mathrm{~nm}^{23}$

There are several techniques that are becoming increasingly popular for studying large areas of two-dimensional (2D)

Received: July 9, 2018

Accepted: August 21, 2018

Published: August 21, 2018 

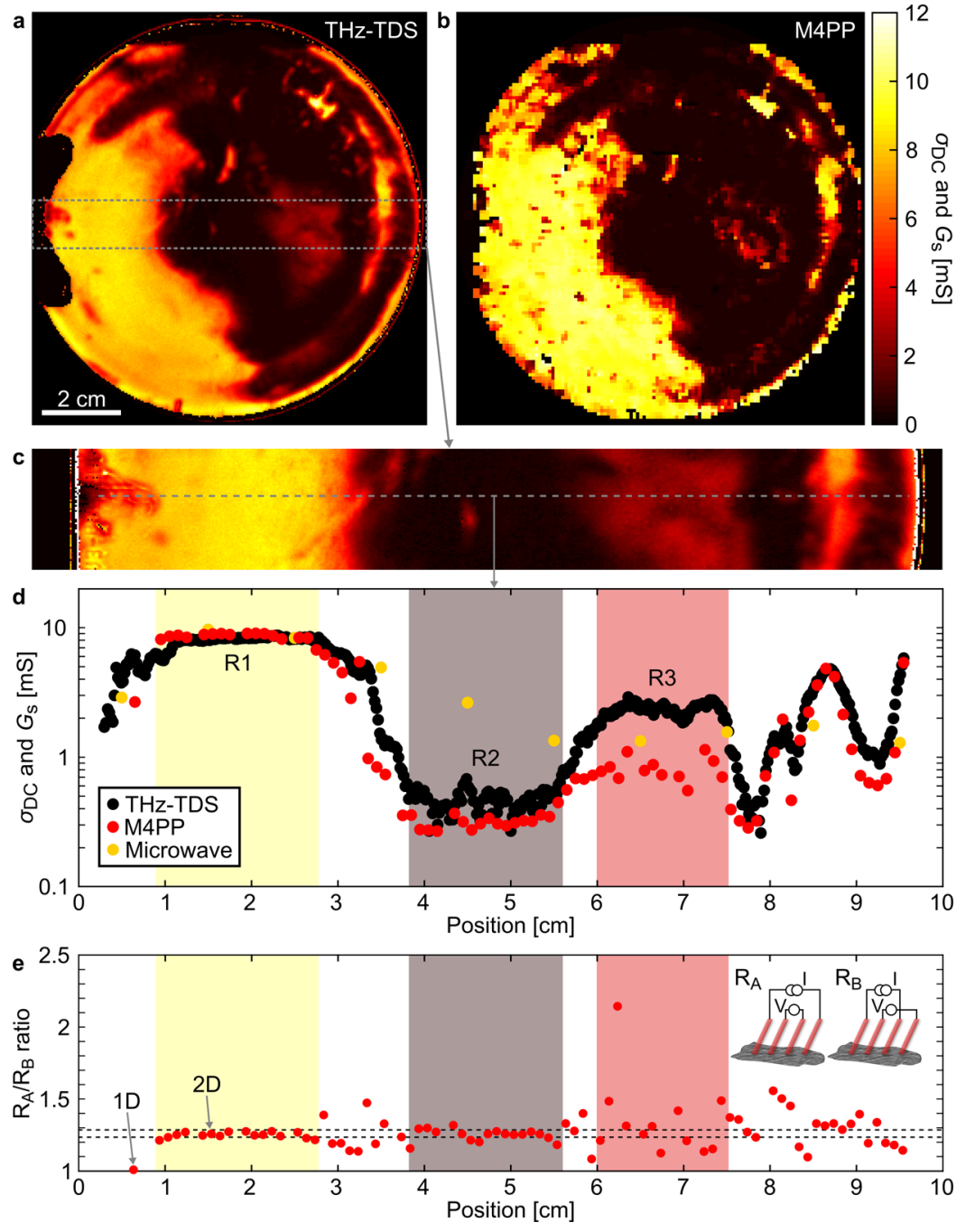

Figure 1. $\sigma_{\mathrm{dc}}$ and $G_{\mathrm{s}}$ map of graphene on a $4 \mathrm{in}$. SiC wafer measured by (a) THz-TDS (400 $\mu \mathrm{m}$ step size) and (b) M4PP (800 $\mu \mathrm{m}$ step size, $10 \mu \mathrm{m}$ probe pitch). (c) THz-TDS map of the central region of the wafer (dotted box in (a)) acquired with $200 \mu \mathrm{m}$ step size. The color bar is the same for $(\mathrm{a}-\mathrm{c})$. The M4PP line scan was acquired along the dotted line $\left(1000 \mu \mathrm{m}\right.$ step size, $350 \mu \mathrm{m}$ probe pitch). (d) Line plot of $\sigma_{\mathrm{dc}}$ and $G_{\mathrm{s}}$ from THzTDS, M4PP, and microwave measurements ( $1 \mathrm{~cm}$ step size) following the line in (c). (e) Line plot of the $R_{\mathrm{A}} / R_{\mathrm{B}}$ resistance ratio for the M4PP line scan shown in (d). The dashed lines indicate $\pm 2.5 \%$ deviation from a $R_{\mathrm{A}} / R_{\mathrm{B}}$ resistance ratio of 1.26 . The insets illustrate the M4PP $R_{\mathrm{A}}$ and $R_{\mathrm{B}}$ configurations.

materials. For instance, terahertz time-domain spectroscopy (THz-TDS), microwave impedance, and micro four-point probe (M4PP) measurements are readily available techniques for mapping the electrical properties of graphene. ${ }^{24-27} \mathrm{THz}-$ TDS and microwave impedance are contactless techniques, both providing information on electrical conductivity. M4PP measures the electrical conductance on scales from micrometer to millimeter depending on the probe distance, using four electrodes that make direct physical contact to the sample surface. In each of the three cases, maps are generated by scanning a given area of the sample.

In this paper, we combine information from the abovementioned electrical and optical mapping techniques and reveal significant variations in electrical properties of QFS 2LG across a 4 in. $\mathrm{SiC}$ wafer, which we attribute to nanoscale structural variations within the $\mathrm{SiC}$ substrate. THz-TDS mapping across the entire wafer reveals order of magnitude variations in the dc sheet conductivity $\left(\sigma_{\mathrm{dc}}\right)$. These results are in excellent agreement with M4PP mapping of the sheet conductance $\left(G_{\mathrm{s}}\right)$. Although these techniques provide a good benchmark, they are limited in spatial resolution to tens or hundreds of micrometers and do not reveal the structural properties of graphene and the substrate. Further employing a host of complementary techniques such as CLSM, Raman spectroscopy, atomic force microscopy (AFM), and Kelvin probe force microscopy (KPFM), we observe three distinct areas of graphene characterized by different electrical properties, which we suggest are formed as a consequence of different levels of defects present in the $\mathrm{SiC}$ substrate prior to the growth, as also evident by the fact that the same areas are found in same locations on different wafers from the same ingot after growth. The findings here show that the structure and quality of $\mathrm{SiC}$ substrates can have drastic impact on the electrical properties of CVD graphene grown on top, even across a single wafer.

\section{RESULTS AND DISCUSSION}

$\sigma_{\mathrm{dc}}$ and $G_{\mathrm{s}}$ of graphene across a $4 \mathrm{in}$. SiC wafer were mapped by $\mathrm{THz}-\mathrm{TDS}$ and $\mathrm{M} 4 \mathrm{PP}$, respectively. The resulting maps (Figure 1a,b) agree well and reveal significant inhomogeneities 

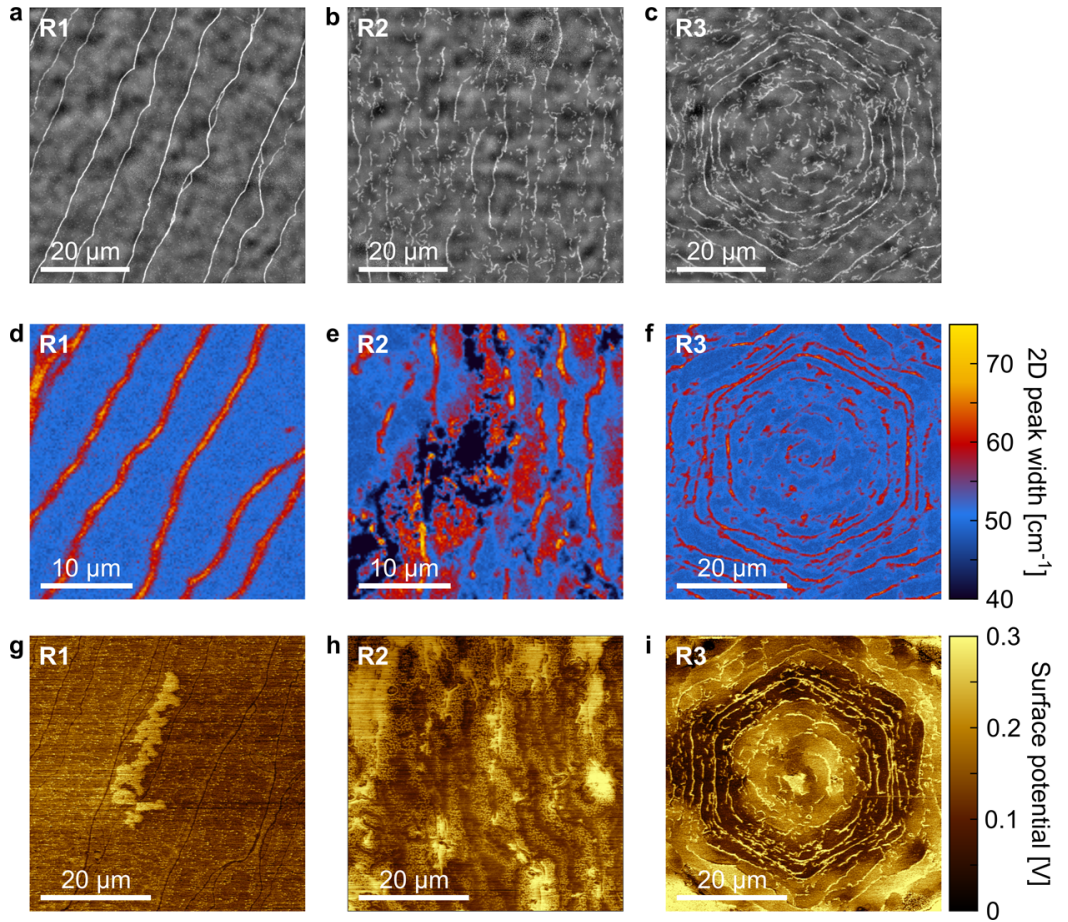

Figure 2. $(a-c)$ CLSM, $(d-f)$ Raman 2D peak width, and (g-i) KPFM surface potential images of graphene on SiC from regions R1, R2, and R3.

in the conducting properties of graphene and highlight variations of more than 1 order of magnitude in $\sigma_{\mathrm{dc}}$ across the 4 in. wafer.

To compare the THz-TDS and M4PP measurements, we performed a higher resolution THz-TDS scan of a stripe across the center of the wafer (Figure 1c). M4PP line scans with larger electrode pitches $(150-450 \mu \mathrm{m})$ were carried out to achieve a measurement probing a length scale similar to that of the THz-TDS measurement. The THz-TDS $\sigma_{\mathrm{dc}}$ values along the same line were extracted. $\sigma_{\mathrm{dc}}$ and $G_{\mathrm{s}}$ from the line scans are shown in Figure 1d and highlight the excellent correlation between the THz-TDS and the M4PP measurements despite both showing variations of a factor of $\sim 30$ across the wafer.

To investigate the reasons behind the variations in $\sigma_{\mathrm{dc}}$ across the 4 in. wafer, we identify three regions of interest (highlighted in Figure 1d) for further studies, R1, R2, and R3. R1 is a region with high and uniform $\sigma_{\mathrm{dc}}$, where the THzTDS and M4PP measurements fully agree. R2 is a region with low and relatively uniform $\sigma_{\mathrm{dc}}$ where the THz-TDS and M4PP measurements also agree. Finally, R3 is a region with intermediate and nonuniform $\sigma_{\mathrm{dc}}$, where the THz-TDS measurements yield a higher $\sigma_{\mathrm{dc}}$ compared to the M4PP measurements.

$G_{s}$ extracted from the M4PP line scan is independent of the probe pitch between 150 and $450 \mu \mathrm{m}$ (Supporting Information Figure S2). Figure 1d also shows the microwave impedance conductivity measured across the same line as the M4PP line scan. $\sigma_{\mathrm{dc}}$ from the microwave impedance measurements agrees quantitatively with the THz-TDS and M4PP measurements in the wide zone R1 but does not track the more rapid spatial variations in $\mathrm{R} 2$ and $\mathrm{R} 3$ because of the far larger spot size (20 $\times 20 \mathrm{~mm}^{2}$ ).

The M4PP $R_{\mathrm{A}} / R_{\mathrm{B}}$ resistance ratio for the line scan is shown in Figure 1e. In regions $\mathrm{R} 1$ and $\mathrm{R} 2$, the current flow is $2 \mathrm{D}$-like $(\sim 1.26)$, whereas the $R_{\mathrm{A}} / R_{\mathrm{B}}$ resistance ratio fluctuates in $\mathrm{R} 3$, indicating that the current flow is more restricted. This also explains why the results from THz-TDS and M4PP deviate in $\mathrm{R} 3$. THz-TDS is sensitive to the nanoscopic conductivity averaged over a length scale corresponding to the characteristic distance traversed by an electron during one cycle of the alternating $\mathrm{THz}$ field, which is on the order of $10-100 \mathrm{~nm} .^{24,25}$ Given that the spot size of the $\mathrm{THz}$ beam is $\sim 350 \mu \mathrm{m}$, the measured values of the conductivity can be considered as the average of all 10-100 $\mathrm{nm}$ interaction regions across the beam spot, which is essentially the averaged conductivity. In contrast, a M4PP measurement with $300 \mu \mathrm{m}$ probe pitch (see illustration in Supporting Information Figure S1) is measuring the $2 \mathrm{D}$ conductance, which is highly sensitive to any obstructions of the current flow, and only identical to the $2 \mathrm{D}$ conductivity for a perfectly uniform conducting film of constant thickness. ${ }^{24,28,29}$

CLSM, KPFM, and Raman spectroscopic mapping were carried out in all three regions, R1, R2, and R3, to further understand the differences in $\sigma_{\mathrm{dc}}$ observed in Figure 1. The CLSM images reveal clear topographical differences between the three regions as shown in Figure $2 a-c$. In region $R 1$, the sample is highly ordered with large flat terraces and welldefined steps. This corresponds well to the $6 \mathrm{H}-\mathrm{SiC}$ bulk crystal in regions where the basal plane is tilted less than $0.03^{\circ}$ from the (0001) direction. This is known to lead to a well-ordered surface morphology from a step-bunching effect during $\mathrm{SiC}$ high-temperature hydrogen etching prior to graphene growth. ${ }^{1,30} \mathrm{R} 2$ exhibits a less-ordered surface structure, where the terraces and steps are not as well-defined as in R1. This is possibly caused by local plane tilting in the $\mathrm{SiC}$ substrate toward various directions. ${ }^{31}$ The surface structure of R3 is different to both the previous regions, this time being highly disordered and showing clear signs of screw dislocations. Here, the hydrogen etching of the $\mathrm{SiC}$ substrate has formed deep pits. ${ }^{32}$ Higher and lower magnification CLSM images from the three regions can be found in the Supporting Information (Figure S3) and show general consistence of images across a 
much larger area. On the basis of CLSM images, R3 can contain up to 100 screw dislocations per $1 \mathrm{~mm}^{2}$ area with the distance between two screw dislocation centers being as low as $\sim 20 \mu \mathrm{m}$.

Maps of the Raman 2D peak width (Figure 2d-f) and the surface potential measured by KPFM (Figure $2 \mathrm{~g}-\mathrm{i}$ ) corroborate well with the features observed in the CLSM images. However, Raman and KPFM also reveal finer structure on the submicron scale. Representative Raman spectra for R1, R2, and R3 are shown in Supporting Information Figure S4 with average values of the $2 \mathrm{D}$ peak widths (full width at halfmaximum) for the three different regions summarized in Supporting Information Table S1. The 2D peak widths of 50.5 $\pm 1.6 \mathrm{~cm}^{-2}$ for the blue regions and $59.7 \pm 3.3 \mathrm{~cm}^{-2}$ for the red/yellow regions in R1 (Figure 2d) are characteristic of 2LG and 3LG, respectively (Supporting Information Figure S4a). ${ }^{22,33}$ The structural quality of $2 \mathrm{LG}$ is highly uniform for R1 with homogeneous $2 \mathrm{LG}$ coverage, where thicker layers of graphene are formed at the step edges. However, the same cannot be said for R2, which shows significant local variations in the 2D peak widths, indicative of nonuniform graphene coverage (Figure 2e). In this region, the average $2 \mathrm{D}$ peak widths are $38.1 \pm 3.7,49.8 \pm 2.0$, and $58.6 \pm 3.1 \mathrm{~cm}^{-2}$, which we assign to $1 \mathrm{LG}, 2 \mathrm{LG}$, and $3 \mathrm{LG}$, respectively (Supporting Information Figure S4b). ${ }^{22,33}$ In R3, we observe a similar 2D peak width of $50.1 \pm 2.1 \mathrm{~cm}^{-2}$ for the blue, 2LG regions, albeit with a slightly larger variation than that of R1 and slightly lower values for red/yellow, 3LG regions, $57.8 \pm 2.2 \mathrm{~cm}^{-2}$ (Supporting Information Figure S4c). Thus, the structural quality of graphene assessed by Raman spectroscopy in R3 (Figure 2f) is somewhere in-between $\mathrm{R} 1$ and $\mathrm{R} 2$.

The surface potential in R1 is highly uniform (except for a contaminant object on the surface with higher surface potential, Figure $2 \mathrm{~g}$ ) as opposed to the more strongly varying surface potential in R2 and R3 (Figure 2h,i). In R2, the KPFM image, similar to the Raman $2 \mathrm{D}$ peak width, shows local surface potential variations across the surface. The screw dislocations in R3 are also visible in the surface potential image, which varies across individual dislocations. The difference in the surface potential at step edges compared to terraces is higher in R3.

Thus, the distinct indications of disrupted current flow in Figure $1 \mathrm{~d}$,e could be explained by the step edges in R3 acting as resistive or reflective barriers obstructing current flow. The step edges are also taller in R3 compared to R1 and R2 (AFM images, Supporting Information Figure S5), which has previously been shown to negatively affect the electrical properties of graphene on $\mathrm{SiC} .{ }^{4-38}$ The AFM images also show that the terraces between steps in the substrate are flat.

From the detailed CLSM, KPFM, and Raman maps obtained from R1, R2, and R3, it seems clear that the profound differences in the structure of the $\mathrm{SiC}$ substrate dictate the growth and corresponding electrical properties of graphene. In R1, we observe a well-ordered topography of the $\mathrm{SiC}$ substrate, which leads to the growth of uniform and highly conducting graphene. A graphene layer with strong variations in the surface potential is found in R2, likely originating from the irregular $\mathrm{SiC}$ surface structure observed here. Disordered graphene found in $\mathrm{R} 2$ has the lowest conductivity across the wafer. In R3, where the THz-TDS and M4PP measurements deviate, we find a high density of screw dislocations with high step edges that seems to affect the current flow. From M4PP measurements conducted inside R3 with a 200 times smaller pitch $(1.5 \mu \mathrm{m})$ than in Figure 1 (Supporting Information Figure S6), we find that the variation in $G_{s}$ across screw dislocations is on the order of the factor of $\sim 30$, as also observed across the full wafer. Such large local variations in $G_{s}$ are bound to lead to variations between THz-TDS (simple spatial average) and M4PP (weighted average between probes) and highlight the complex nature of graphene grown across screw dislocations in R3. Interestingly, the M4PP measurements with small $1.5 \mu \mathrm{m}$ probe pitch, shown in Supporting Information Figure S6, highlight that $G_{s}$ in R3 actually contains the same two conductance plateaus as in R1 and R2 and that the intermediate conductivity measured by THz-TDS and M4PP with large electrode pitch in R3 is an average of these. The difference between the measurements originates from the spatial distribution of current flow between electrodes in M4PP measurements being greatly perturbed, whereas the THz-TDS measurement is affected less because it averages over smaller interaction regions across the beam spot.

The claim that the variation in electrical properties of graphene is due to pre-existing defects in the $\mathrm{SiC}$ substrate is backed up by a THz-TDS measurement performed on graphene, which was grown on another $\mathrm{SiC}$ wafer from the same ingot as the $\mathrm{SiC}$ wafer shown in Figure 1. The $\sigma_{\mathrm{dc}}$ map of the second wafer (Supporting Information Figure S7) shows features that are very similar to the map of the initial wafer. The $\sigma_{\mathrm{dc}}$ values are lower for the second wafer, but it clearly shows regions with high and low conductivity that are spatially located at the same areas on the two wafers, thereby supporting the notion that the surface of the $\mathrm{SiC}$ substrate is of utmost importance for achieving a homogeneous graphene growth.

\section{CONCLUSIONS}

THz-TDS and M4PP measurements were performed to map the electrical properties of QFS graphene across a $4 \mathrm{in}$. SiC wafer. The two measurement techniques are in good agreement and demonstrate how $\sigma_{\mathrm{dc}}$ of the QFS graphene can vary by a factor of $\sim 30$ across a wafer. Three distinct regions with specific $\sigma_{\mathrm{dc}}$ behavior were observed. From CLSM, KPFM, and Raman spectroscopy, we find that the differences in electrical properties across the wafer can be attributed mainly to variations in the $\mathrm{SiC}$ surface structure that are present prior to graphene growth. The substrate quality, therefore, has a profound impact on the resulting graphene growth. It was found that more defect-free $\mathrm{SiC}$ surfaces yield graphene with higher conductivity and with more uniform current flow. The results revealed here give an increased understanding on the crucial importance of the substrate for high-quality growth of graphene on SiC. Additionally, THzTDS can be used for optimizing the conditions of the graphene growth regardless of the quality of the $\mathrm{SiC}$ substrate and for rapidly prescanning of as-grown or QFS graphene samples to identify areas with optimal properties for specific applications.

\section{METHODS}

Semi-insulating chemomechanically polished $\mathrm{SiC}$ substrates were hydrogen-etched prior to graphene growth to remove a few tens of nanometers of the upper $\mathrm{SiC}$, which may have been damaged in the polishing procedure. Etching was performed at $1600{ }^{\circ} \mathrm{C}$ and 100 mbar. Epitaxial graphene was subsequently grown by $\mathrm{CVD} /$ vapor phase epitaxy on the Si-face of nominally on-axis $6 \mathrm{H}-\mathrm{SiC}$ in a hot-wall Aixtron G5 reactor commercially used for $\mathrm{SiC}$ epi-growth under similar conditions as applied for hydrogen etching, that is, $T=1600$ 
${ }^{\circ} \mathrm{C}$ and $P=100$ mbar. Carbon atoms were provided externally by methane delivered to the reactor by an argon carrier gas and deposited (with epitaxy) on the $\mathrm{SiC}$ substrate. This method offers the precision of synthesizing a predefined number of carbon layers, including a single layer on the Si-face of $\mathrm{SiC}^{1,39}$ With CVD, the nucleation sites for graphene growth are located at the atomic steps, therefore enabling step-flow epitaxy. More details on the growth process can be found in the Supporting Information. The samples were intercalated with hydrogen after growth to decouple graphene from the $\mathrm{SiC}$ substrate and obtain QFS graphene.,12

THz-TDS measurements were conducted using a commercial fibercoupled Picometrix spectrometer. ${ }^{25}$ The sample was scanned in the focal plane of a $\mathrm{THz}$ beam at normal incidence to form a spatial map with spatial resolution given by the diffraction-limited spot size of $\sim 350 \mu \mathrm{m}$ at $1 \mathrm{THz}$ (Supporting Information Figure S1). The frequency-dependent sheet conductivity of graphene, $\sigma_{\mathrm{s}}(\omega)=\sigma_{1}+$ $\mathrm{i} \sigma_{2}$, can be determined for each pixel in the raster-scanned map through the transmission function $T_{\text {film }}(\omega)=E_{\text {film }}(\omega) / E_{\text {ref }}(\omega)$, where $E_{\text {film }}$ and $E_{\text {ref }}$ are the Fourier transforms of the $\mathrm{THz}$ waveforms transmitted through graphene-covered $\mathrm{SiC}$ and nongraphene-covered $\mathrm{SiC}$, respectively. The frequency-dependent sheet conductivity is extracted from the transmission function as ${ }^{40}$

$$
\sigma_{\mathrm{s}}(\omega)=\frac{n+1}{Z_{0}}\left(\frac{1}{T_{\text {film }}(\omega)}-1\right)
$$

where $Z_{0}$ is the vacuum impedance and $n$ is the refractive index of the $\mathrm{SiC}$ substrate. ${ }^{41} \sigma_{\mathrm{dc}}$ is extracted, as described previously, ${ }^{42}$ by fitting the real part of $\sigma_{\mathrm{s}}(\omega)$ from 0.4 to $1.1 \mathrm{THz}$ to the Drude model, $\sigma_{1}(\omega)$ $=\sigma_{\mathrm{dc}} /(1-\mathrm{i} \omega \tau)$, where $\tau$ is the carrier scattering time.

M4PP mapping of $G_{s}$ was performed on a semiautomatic CAPRES microRSP-M300 using $10 \mu \mathrm{m}$ electrode pitch, lock-in technique (12 $\mathrm{Hz}$ ), and a set current of $250 \mu \mathrm{A}$. The surface was detected during engagement using a strain gauge sensor to maintain consistent probe pressure and avoid sample damage. ${ }^{43}$ Measurements were conducted in two configurations (labeled $\mathrm{A}$ and $\mathrm{B}$, see inset in Figure 1e). From the M4PP resistance ratio $\left(R_{\mathrm{A}} / R_{\mathrm{B}}\right)$, it is possible to determine whether the current flow in the sample is qualitatively one- or twodimensional ( $1 \mathrm{D}$ or $2 \mathrm{D}$ ). If $R_{\mathrm{A}} / R_{\mathrm{B}}=1$, the current flow is $1 \mathrm{D}$-like, typical for damaged regions where the source-drain current flow is restricted to narrow pathways, whereas $R_{\mathrm{A}} / R_{\mathrm{B}} \approx 1.26$ corresponds to an uninhibited current flow, where the sample is homogeneously conducting in two dimensions. ${ }^{28,29}$ The M4PP measurements were conducted with the probes aligned horizontally in all images shown as indicated by the probe positions in Supporting Information Figure S1.

Microwave impedance measurements were carried out to measure the sheet resistivity $\left(R_{\mathrm{s}}\right)$ of the graphene film. ${ }^{44} R_{\mathrm{s}}$ was determined by measuring the shifts induced to the frequency and quality factor of a sapphire microwave resonator located inside of an electromagnetic cavity. During the measurement, the sample is placed over a $20 \times 20$ $\mathrm{mm}^{2}$ opening of the cavity, from which the evanescent near-field emerges. The carriers in graphene interact with the electromagnetic field, thereby shifting the resonance of sapphire, which is measured using a vector network analyzer. $R_{\mathrm{s}}$ can be determined by taking into account the substrate thickness $\left(t_{\mathrm{s}}\right)$, permittivity of free space $\left(\epsilon_{0}\right)$, relative permittivity of $\mathrm{SiC}\left(\epsilon_{\mathrm{SiC}}\right)$, shift in linewidth due to substrate $\left(\Delta w_{\mathrm{s}}\right)$ and graphene $\left(\Delta w_{\mathrm{g}}\right)$, and frequency of the resonator $\left(f_{0}\right)^{45}$

$$
R_{\mathrm{s}}=\frac{\Delta f}{\pi f_{0} \epsilon_{0} t_{\mathrm{s}}\left(\epsilon_{\mathrm{SiC}}-1\right)\left(\Delta w_{\mathrm{g}}-\Delta w_{\mathrm{s}}\right)}
$$

CLSM was performed using an Olympus LEXT OLS4000 system in ambient air. ${ }^{23}$ It is designed to operate in reflection mode, and the optical image of the sample was produced by using a photomultiplier tube to capture the reflected optical signal from the $405 \mathrm{~nm}$ wavelength laser that is scanned across the sample surface using a micro-electro-mechanical system scanner and galvano mirrors. The system uses a confocal pinhole to eliminate out-of-focus light, which enhances the spatial resolution of the system to $\sim 150 \mathrm{~nm}$. However, using the confocal setup significantly reduces the depth-of-field as the sample plane is often not perpendicular to the optical axis of the microscope objective, with only a small portion of the field-of-view being in focus at a time. A fully in-focus image is achieved by capturing a series of images at different focal-planes along the $z$-axis (minimum of $60 \mathrm{~nm}$ steps) and combining the brightest pixels from each z-stack. This often leads to artifacts, where neighboring brightest pixels from adjacent $\mathrm{z}$-stacks have different brightest intensities.

Raman spectroscopy was performed using a Renishaw InVia system fitted with a $532 \mathrm{~nm}$ excitation laser and 1800 groves/mm grating. The laser is focused to approximately $800 \mathrm{~nm}$ spot size through a $100 \times$ objective $(0.85 \mathrm{NA})$, and the confocality was set to $65 \mu \mathrm{m}$ slit opening. The maps were acquired in ambient air with $100 \mathrm{~nm}$ pixel size, $0.25 \mathrm{~s}$ exposure, and $\sim 10 \mathrm{~mW}$ laser power. The maps were generated by first normalizing to the highest $\mathrm{SiC}$ peak and then subtracting a reference $\mathrm{SiC}$ spectra. The $\mathrm{G}$ - and $2 \mathrm{D}$-peaks of the resulting spectra were fitted to extract maps for the area, intensity, width, and shift. ${ }^{22}$

Topography (AFM) and surface potential measurements were performed using the Bruker Icon scanning probe microscope in ambient air. The measurements were carried out using a two-pass mode. During the first pass, the topography of the sample was mapped using PeakForce Tapping with PFQNE-AL probes. The PeakForce mode performs force-curves at a rate of $2 \mathrm{kHz}$ and extracts the topography at the specified set point force of $5 \mathrm{nN}^{46}$ In the second pass, the surface potential of the sample was mapped using frequency-modulated KPFM implementation. During the second pass, the probe is kept at a constant lift height of $45 \mathrm{~nm}$ by tracing the topography line obtained during the first pass. To measure the surface potential (commonly referred to as the contact potential difference, $\left.V_{\mathrm{CPD}}\right)$, the cantilever is driven on resonance $\left(f_{0}\right)$ while a lowfrequency $\left(f_{\text {mod }}\right)$ ac voltage $\left(V_{\mathrm{ac}}\right)$ is applied to the conductive probe. This externally applied voltage, together with any other inherent potential differences between the probe and sample, leads to electrostatic attraction/repulsion that shifts the resonance of the cantilever by $f_{0} \pm f_{\text {mod }}$ and other higher harmonics of $f_{\text {mod. }}$. The surface potentials are mapped by using a PID feedback that monitors these side lobes and minimizes them by applying an additional $\mathrm{dc}$ voltage $\left(V_{\mathrm{dc}}\right)$ such that $V_{\mathrm{dc}}=V_{\mathrm{CPD}}$, thus compensating for $V_{\mathrm{CPD}}$ and measuring it at the same time. Further details on FM-KPFM are available elsewhere. ${ }^{47}$

\section{ASSOCIATED CONTENT}

\section{S Supporting Information}

The Supporting Information is available free of charge on the ACS Publications website at DOI: 10.1021/acsami.8b11428.

Schematic representation of measurements; M4PP line scan data; CLSM images; Raman spectra and 2D peak widths; AFM characterization; THz map of the second $\mathrm{SiC}$ wafer; and details on graphene growth (PDF)

\section{AUTHOR INFORMATION}

\section{Corresponding Author}

*E-mail: peter.boggild@nanotech.dtu.dk.

ORCID

Patrick R. Whelan: 0000-0002-3978-7029

Christos Melios: 0000-0003-3890-5457

Peter Bøggild: 0000-0002-4342-0449

\section{Notes}

The authors declare no competing financial interest.

\section{ACKNOWLEDGMENTS}

This work was supported by the Danish National Research Foundation (DNRF) Center for Nanostructured Graphene (DNRF103), EU Graphene Flagship Core 1 (grant agreement: 696656), EU Graphene Flagship Core 2 (grant agreement: 
785219), EU Horizon 2020 (3DAM, grant agreement: 692527), 16NRM01 GRACE, and the Department for Business, Energy and Industrial Strategy though NMS funding.

\section{REFERENCES}

(1) Strupinski, W.; Grodecki, K.; Wysmolek, A.; Stepniewski, R.; Szkopek, T.; Gaskell, P. E.; Grüneis, A.; Haberer, D.; Bozek, R.; Krupka, J.; Baranowski, J. M. Graphene Epitaxy by Chemical Vapor Deposition on SiC. Nano Lett. 2011, 11, 1786-1791.

(2) Tokarczyk, M.; Kowalski, G.; Możdżonek, M.; Borysiuk, J.; Stępniewski, R.; Strupiński, W.; Ciepielewski, P.; Baranowski, J. M. Structural Investigations of Hydrogenated Epitaxial Graphene Grown on 4H-SiC (0001). Appl. Phys. Lett. 2013, 103, 241915.

(3) Hwang, J.; Shields, V. B.; Thomas, C. I.; Shivaraman, S.; Hao, D.; Kim, M.; Woll, A. R.; Tompa, G. S.; Spencer, M. G. Epitaxial Growth of Graphitic Carbon on C-Face $\mathrm{SiC}$ and Sapphire by Chemical Vapor Deposition (CVD). J. Cryst. Growth 2010, 312, 3219-3224.

(4) Michon, A.; Vézian, S.; Ouerghi, A.; Zielinski, M.; Chassagne, T.; Portail, M. Direct Growth of Few-Layer Graphene on $6 \mathrm{H}-\mathrm{SiC}$ and 3C-SiC/Si via Propane Chemical Vapor Deposition. Appl. Phys. Lett. 2010, 97, 171909.

(5) Liu, Q.; Yu, C.; He, Z.; Gu, G.; Wang, J.; Zhou, C.; Guo, J.; Gao, X.; Feng, Z. Chemical Vapor Deposition Graphene of High Mobility by Gradient Growth Method on an 4H-SiC $\left(\begin{array}{llll}0 & 0 & 0 & 1\end{array}\right)$ Substrate. Appl. Surf. Sci. 2018, 454, 68-73.

(6) Riedl, C.; Coletti, C.; Iwasaki, T.; Zakharov, A. A.; Starke, U. Quasi-Free-Standing Epitaxial Graphene on $\mathrm{SiC}$ Obtained by Hydrogen Intercalation. Phys. Rev. Lett. 2009, 103, 246804.

(7) Tian, H.; Yang, Y.; Xie, D.; Cui, Y.-L.; Mi, W.-T.; Zhang, Y.; Ren, T.-L. Wafer-Scale Integration of Graphene-Based Electronic, Optoelectronic and Electroacoustic Devices. Sci. Rep. 2015, 4, 3598.

(8) Habibpour, O.; He, Z. S.; Strupinski, W.; Rorsman, N.; Zirath, H. Wafer Scale Millimeter-Wave Integrated Circuits Based on Epitaxial Graphene in High Data Rate Communication. Sci. Rep. 2017, 7, 41828 .

(9) Winters, M.; Sveinbjörnsson, E. Ö.; Melios, C.; Kazakova, O.; Strupiński, W.; Rorsman, N. Characterization and Physical Modeling of MOS Capacitors in Epitaxial Graphene Monolayers and Bilayers on 6H-SiC. AIP Adv. 2016, 6, 085010.

(10) Cagliani, A.; Mackenzie, D. M. A.; Tschammer, L. K.; Pizzocchero, F.; Almdal, K.; Bøggild, P. Large-Area Nanopatterned Graphene for Ultrasensitive Gas Sensing. Nano Res. 2014, 7, 743754.

(11) Varghese, S. S.; Lonkar, S.; Singh, K. K.; Swaminathan, S.; Abdala, A. Recent Advances in Graphene Based Gas Sensors. Sens. Actuators, B 2015, 218, 160-183.

(12) Melios, C.; Winters, M.; Strupiński, W.; Panchal, V.; Giusca, C. E.; Jayawardena, K. D. G. I.; Rorsman, N.; Silva, S. R. P.; Kazakova, O. Tuning Epitaxial Graphene Sensitivity to Water by Hydrogen Intercalation. Nanoscale 2017, 9, 3440-3448.

(13) Bonaccorso, F.; Sun, Z.; Hasan, T.; Ferrari, A. C. Graphene Photonics and Optoelectronics. Nat. Photonics 2010, 4, 611-622.

(14) Yang, X.; Vorobiev, A.; Generalov, A.; Andersson, M. A.; Stake, J. A Flexible Graphene Terahertz Detector. Appl. Phys. Lett. 2017, $111,021102$.

(15) Yan, H.; Xia, F.; Zhu, W.; Freitag, M.; Dimitrakopoulos, C.; Bol, A. A.; Tulevski, G.; Avouris, P. Infrared Spectroscopy of WaferScale Graphene. ACS Nano 2011, 5, 9854-9860.

(16) Bae, S.-H.; Zhou, X.; Kim, S.; Lee, Y. S.; Cruz, S. S.; Kim, Y.; Hannon, J. B.; Yang, Y.; Sadana, D. K.; Ross, F. M.; Park, H.; Kim, J. Unveiling the Carrier Transport Mechanism in Epitaxial Graphene for Forming Wafer-Scale, Single-Domain Graphene. Proc. Natl. Acad. Sci. U. S. A. 2017, 114, 4082-4086.

(17) Yager, T.; Lartsev, A.; Yakimova, R.; Lara-Avila, S.; Kubatkin, S. Wafer-Scale Homogeneity of Transport Properties in Epitaxial Graphene on SiC. Carbon 2015, 87, 409-414.
(18) Koon, D. W.; Wang, F.; Petersen, D. H.; Hansen, O. Sensitivity of Resistive and Hall Measurements to Local Inhomogeneities. J. Appl. Phys. 2013, 114, 163710.

(19) Koon, D. W.; Wang, F.; Petersen, D. H.; Hansen, O. Sensitivity of Resistive and Hall Measurements to Local Inhomogeneities: FiniteField, Intensity, and Area Corrections. J. Appl. Phys. 2014, 116, 133706.

(20) Mackenzie, D. M. A.; Buron, J. D.; Whelan, P. R.; Caridad, J. M.; Bjergfelt, M.; Luo, B.; Shivayogimath, A.; Smitshuysen, A. L.; Thomsen, J. D.; Booth, T. J.; Gammelgaard, L.; Zultak, J.; Jessen, B. S.; Bøggild, P.; Petersen, D. H. Quality Assessment of Graphene: Continuity, Uniformity, and Accuracy of Mobility Measurements. Nano Res. 2017, 10, 3596-3605.

(21) Jessen, B. S.; Whelan, P. R.; Mackenzie, D. M. A.; Luo, B.; Thomsen, J. D.; Gammelgaard, L.; Booth, T. J.; Bøggild, P. Quantitative Optical Mapping of Two-Dimensional Materials. Sci. Rep. 2018, 8, 6381.

(22) Melios, C.; Panchal, V.; Giusca, C. E.; Strupiński, W.; Silva, S. R. P.; Kazakova, O. Carrier Type Inversion in Quasi-Free Standing Graphene: Studies of Local Electronic and Structural Properties. Sci. Rep. 2015, 5, 10505.

(23) Panchal, V.; Yang, Y.; Cheng, G.; Hu, J.; Kruskopf, M.; Liu, C.I.; Rigosi, A. F.; Melios, C.; Walker, A. R. H.; Newell, D. B.; Kazakova, O.; Elmquist, R. E. Confocal Laser Scanning Microscopy: A Tool for Rapid Optical Characterization of 2D Materials. arXiv 1804.04420, 2018.

(24) Bøggild, P.; Mackenzie, D. M. A.; Whelan, P. R.; Petersen, D. H.; Buron, J. D.; Zurutuza, A.; Gallop, J.; Hao, L.; Jepsen, P. U. Mapping the Electrical Properties of Large-Area Graphene. 2D Mater. 2017, 4, 042003.

(25) Buron, J. D.; Petersen, D. H.; Bøggild, P.; Cooke, D. G.; Hilke, M.; Sun, J.; Whiteway, E.; Nielsen, P. F.; Hansen, O.; Yurgens, A.; Jepsen, P. U. Graphene Conductance Uniformity Mapping. Nano Lett. 2012, 12, 5074-5081.

(26) Mackenzie, D. M. A.; Whelan, P. R.; Bøggild, P.; Jepsen, P. U.; Redo-Sanchez, A.; Etayo, D.; Fabricius, N.; Petersen, D. H. Quality Assessment of Terahertz Time-Domain Spectroscopy Transmission and Reflection Modes for Graphene Conductivity Mapping. Opt. Express 2018, 26, 9220-9229.

(27) Klarskov, M. B.; Dam, H. F.; Petersen, D. H.; Hansen, T. M.; Löwenborg, A.; Booth, T. J.; Schmidt, M. S.; Lin, R.; Nielsen, P. F.; Bøggild, P. Fast and Direct Measurements of the Electrical Properties of Graphene Using Micro Four-Point Probes. Nanotechnology 2011, 22,445702

(28) Lotz, M. R.; Boll, M.; Hansen, O.; Kjær, D.; Bøggild, P.; Petersen, D. H. Revealing Origin of Quasi-One Dimensional Current Transport in Defect Rich Two Dimensional Materials. Appl. Phys. Lett. 2014, 105, 053115.

(29) Lotz, M. R.; Boll, M.; Østerberg, F. W.; Hansen, O.; Petersen, D. H. Mesoscopic Current Transport in Two-Dimensional Materials with Grain Boundaries: Four-Point Probe Resistance and Hall Effect. J. Appl. Phys. 2016, 120, 134303.

(30) Giannazzo, F.; Deretzis, I.; Nicotra, G.; Fisichella, G.; Ramasse, Q. M.; Spinella, C.; Roccaforte, F.; La Magna, A. High resolution study of structural and electronic properties of epitaxial graphene grown on off-axis 4H-SiC (0001). J. Cryst. Growth 2014, 393, 150155.

(31) Dimitrakopoulos, C.; Grill, A.; McArdle, T. J.; Liu, Z.; Wisnieff, R.; Antoniadis, D. A. Effect of SiC Wafer Miscut Angle on the Morphology and Hall Mobility of Epitaxially Grown Graphene. Appl. Phys. Lett. 2011, 98, 222105.

(32) Hannon, J. B.; Tromp, R. M. Pit Formation during Graphene Synthesis on SiC(0001): In Situ Electron Microscopy. Phys. Rev. B: Condens. Matter Mater. Phys. 2008, 77, 241404.

(33) Grodecki, K.; Jozwik, I.; Baranowski, J. M.; Teklinska, D.; Strupinski, W. SEM and Raman Analysis of Graphene on SiC(0001). Micron 2016, 80, 20-23.

(34) Yager, T.; Lartsev, A.; Mahashabde, S.; Charpentier, S.; Davidovikj, D.; Danilov, A.; Yakimova, R.; Panchal, V.; Kazakova, O.; 
Tzalenchuk, A.; Lara-Avila, S.; Kubatkin, S. Express Optical Analysis of Epitaxial Graphene on SiC: Impact of Morphology on Quantum Transport. Nano Lett. 2013, 13, 4217-4223.

(35) Nicotra, G.; Ramasse, Q. M.; Deretzis, I.; La Magna, A.; Spinella, C.; Giannazzo, F. Delaminated Graphene at Silicon Carbide Facets: Atomic Scale Imaging and Spectroscopy. ACS Nano 2013, 7, 3045-3052.

(36) Ji, S.-H.; Hannon, J. B.; Tromp, R. M.; Perebeinos, V.; Tersoff, J.; Ross, F. M. Atomic-Scale Transport in Epitaxial Graphene. Nat. Mater. 2012, 11, 114-119.

(37) Hollander, M. J.; Agrawal, A.; Bresnehan, M. S.; LaBella, M.; Trumbull, K. A.; Cavalero, R.; Snyder, D. W.; Datta, S.; Robinson, J. A. Heterogeneous Integration of Hexagonal Boron Nitride on Bilayer Quasi-Free-Standing Epitaxial Graphene and Its Impact on Electrical Transport Properties. Phys. Status Solidi A 2013, 210, 1062-1070.

(38) Bryan, S. E.; Yang, Y.; Murali, R. Conductance of Epitaxial Graphene Nanoribbons: Influence of Size Effects and Substrate Morphology. J. Phys. Chem. C 2011, 115, 10230-10235.

(39) Strupinski, W. Method of Graphene Manufacturing. U.S. Patent 9,067,796 B2, 2010.

(40) Buron, J. D.; Pizzocchero, F.; Jessen, B. S.; Booth, T. J.; Nielsen, P. F.; Hansen, O.; Hilke, M.; Whiteway, E.; Jepsen, P. U.; Bøggild, P.; Petersen, D. H. Electrically Continuous Graphene from Single Crystal Copper Verified by Terahertz Conductance Spectroscopy and Micro Four-Point Probe. Nano Lett. 2014, 14, 6348-6355.

(41) Strait, J. H.; George, P. A.; Dawlaty, J.; Shivaraman, S.; Chandrashekhar, M.; Rana, F.; Spencer, M. G. Emission of Terahertz Radiation from SiC. Appl. Phys. Lett. 2009, 95, 051912.

(42) Buron, J. D.; Mackenzie, D. M. A.; Petersen, D. H.; Pesquera, A.; Centeno, A.; Bøggild, P.; Zurutuza, A.; Jepsen, P. U. Terahertz Wafer-Scale Mobility Mapping of Graphene on Insulating Substrates without a Gate. Opt. Express 2015, 23, 30721.

(43) Petersen, D. H.; Hansen, O.; Hansen, T. M.; Bøggild, P.; Lin, R.; Kjær, D.; Nielsen, P. F.; Clarysse, T.; Vandervorst, W.; Rosseel, E.; Bennett, N. S.; Cowern, N. E. B. Review of Electrical Characterization of Ultra-Shallow Junctions with Micro Four-Point Probes. J. Vac. Sci. Technol., B: Nanotechnol. Microelectron.: Mater., Process., Meas., Phenom. 2010, 28, C1C27-C1C33.

(44) Krupka, J.; Strupinski, W. Measurements of the Sheet Resistance and Conductivity of Thin Epitaxial Graphene and SiC Films. Appl. Phys. Lett. 2010, 96, 082101.

(45) Hao, L.; Chen, J.; Liu, Q.; Gallop, J. Microwave Method for High-Frequency Properties of Graphene. IET Circuits, Devices Syst. 2015, 9, 397-402.

(46) Pittenger, B.; Erina, N.; Su, C. Mechanical Property Mapping at the Nanoscale Using PeakForce QNM Scanning Probe Technique. In Nanomechanical Analysis of High Performance Materials; Solid Mechanics and its Applications; Tiwari, A., Ed.; Springer: Dordrecht, 2014; pp 31-51.

(47) Panchal, V.; Pearce, R.; Yakimova, R.; Tzalenchuk, A.; Kazakova, O. Standardization of Surface Potential Measurements of Graphene Domains. Sci. Rep. 2013, 3, 2597. 